\title{
Über das Parachinanisol.
}

\author{
Von Zd. H. Skraup.
}

(Aus dem Laboratorium der Wiener Handelsakademie XVII.)

(Vorgelegt in der Sitzung am 16. Juli 1885.)

Das Parachinanisol oder der Paraoxychinolinmethyläther steht zum Chinin (und Chinidin) in sehr naher Beziehung.

Die durch Oxydation der zwei genannten Chinaalkaloide entstehende Chininsäure $\mathrm{C}_{11} \mathrm{H}_{9} \mathrm{NO}_{3}$ ist ja als Carbonsäure des Paraoxychinolinmethyläthers erkannt worden. ${ }^{1}$

Eine eingehendere Untersuchung des letzteren konnte desshalb in chemischer und physiologischer Beziehung von Nutzen sein, einmal beitragen die chemische Constitution des Chinins und Chinidin weiter kennen zu lernen, dann Anhaltspunkte über die physiologisehe Wirkung der zwei genannten Heilmittel geben, vielleicht auch zu Körpern führen, denen selbst hervorragende physiologische Wirkung eigen ist.

Das Parachinanisol ist, und zwar durch Methylirung des $p$-Oxychinolins mit Jodmethyl und Ätzkali schon dargestellt und flüchtig beschrieben worden. ${ }^{2}$ Diese Darstellungsmethode lässt aber Vieles zu wünschen übrig, und war es daher nothwendig, eine bequemere und quantitativ befriedigendere $z u$ finden, bei der das schwierig rein darstellbare $p$-Oxychinolin ausser Betracht bleibt.

Nachdem, wie ich beim Orthooxychinolin beschrieben habe ${ }^{3}$, der Methyläther desselben entsteht, wenn das Orthoanisidin der Glycerinreaction unterzogen wird, war voraussichtlich, dass Paranisidin das Parachinanisol geben müsste und handelte es sich eigentlich hauptsächlich darum, die glatte Überführung von Paranitrophenol, das durch Nitrirung von Phenol in reichlicher

I Skraup, Wien. Monatshefte II, 587 .

2 Skraup, ebend. III, 544.

3 Skraup, ebénd. III, 557. 
Menge erhalten werden kann, in Nitranisol und die Reduction des letzteren in das Anisidin ohne Schwierigkeiten zn bewerkstelligen. Das Paranitranisol hat zuerst Brunck durch Erhitzen des Phenolsilbersalzes mit Jodmethyl im verschlossenen Rohr dargestellt. Weit bequemer kann man es dadurch erhalten, dass man eine methylalkoholische Lösung des Nitrophenols mit je 1 Mol. Ätzkali und Jodmethyl etwa 18 Stunden auf $100^{\circ}$ erhitzt. (Bei Atmosphärendruck ist kaum Einwirkung zu beobachtem.)

Nach dem Erkalten ist ein Theil des Äthers dem auskrystallisirten Jodkalium beigemengt, und von diesem durch Umschmelzen in wenig Wasser leicht zu trennen; der grössere Antheil ist in alkoholischer Lösung und wird nach dem Abdestilliren des Holzgeistes als braunes ÖI erhalten, das mit Wasserdampf ïbergetrieben wird.

Die Reduction des Nitranisols gelingt in bekannter Weise mit Zinn und Salzsänre ohne Schwierigkeit und quantitativ, vorausgesetzt, dass sie nicht zu stürmisch verläuft. Das Anisidin wird am leichtesten dadurch frei gemacht, dass man die Reductionsflüssigkeit stark eindampft, das nach dem Erkalten quantitativ auskrystallisirte Zinnchloridsalz absaugt und in etwas iuberschüssige concentrirte Kalilauge unter gelindem Erwärmen einträgt. Das ölig abgeschiedene Anisidin erstarrt in der Kälte leicht zu fast reinweissen Krystallen, ist dann zu weiterer Verarbeitung auf Chinanisol genïgend, nach einmaligem Umkrystal. lisiren aus heissem Wasser vollständig rein.

\section{Darstellung von Parachinanisol.}

Das $p$-Anisidin geht beim Erhitzen mit Glycerin und Schwefelsäure in Chinanisol iiber, ob gleichzeitig Nitranisol oder Nitrobenzol zugesetzt ist, ohne dass die Ausbeute sich merklich ändert. In der Regel habe ich ersteres verwendet.

Dagegen ist für das Gelingen des Processes die Menge der zugesetzten Schwefelsäure von grosser Wichtigkeit. Bei kleineren Mengen ist die Verwandlung des Anisidins sehr unvollständig bei zu grossen tritt theilweise Verharzung ein und in beiden Fällen hat die Reinigung des Productes manche Schwierigkeiten. 
Recht befriedigende Ausbeute errielt man unter folgenden Verhältnissen:

78 Grm. Anisidin, 50 Grm. Nitranisol, 320 Grm. Glycerin und 125 Grm. englische Schwefelsäure werden vor dem Rückflusskühler erhitzt. Die eintretende Reaction ist kräftig, doch nie so stiurmisch, dass Kühlung des Kolbens nothwendig wäre. Nach zweistündigem Erhitzen fügt man vorsichtig noch 50 Grm. Schwefelsäure zu und erhitzt noch weitere zwei Stunden. Die mit Wasser verdünnte Reactionsfluissigkeit scheidet mit Wasser verdünnt unverändertes Nitranisol ab (20 - 25 Grm.), der Rest (2-3 Grm.) geht beim Einblasen von Dampf mit diesem über.

Der saure Destillationsribckstand scheidet auf Zusatz von Natronlauge oder Ammoniak freies Chinanisol und unverändertes Anisidin $a b$, die durch Wasserdampf abgetrieben oder mit $\ddot{A} t h e r$ ausgeschüttelt werden.

Die Trennung beider ist in verschiedener Weise möglich. Handelt es sich hauptsächlich um Reindarstellung des Chinanisols und ist der Verlust des Anisidins gleichgiltig, so ist Kaliumbichromat anwendbar, das der verdünnt salzsauren Lösung des Basengemisches im Überschusse zugefügt, ein je nach Umständen gelbes bis braunes Chromat des Chinanisols niederschlägt, während das Anisidin respective dessen Oxydationsprodncte vorwiegend in der violetten Lösung bleiben.

Der hieraus erwachsende Verlust entfällt, wenn die Basen im Wege der sauren Sulfate getrennt werden, von denen das des Chinanisols in Alkohol schwer, das des Anisidina weit leichter löslich ist. Es genügt hiezu die durch wiederholtes Ausschïtteln mit $\ddot{A}$ ther erhaltenen Basen nach Abdestilliren des Äthers in etwa dem $1 \frac{1}{2}$ fachen Volum Alkohol zu lösen, die annähernd berechnete Menge concentrirter Schwefelsäure zuzufügen, um eine nahezu ungefärbte Krystallisation zu erhalten, die mit Alkohol gewaschen weder mit Chromsäure noch Eisenchlorid die Anisidinreaction zeigt.

$60 \mathrm{Grm}$. Rohbase gaben derart behandelt $90 \mathrm{Grm}$. Sulfat, das ist etwa 95\% der theoretischen Ausbeute.

Es wurden noch andere Trennungsmethoden versucht, so unter anderem die vermittelst der Salzsänreverbindungen, von denen die des Anisidins in Salzsäure fast unlöslich, die des Parachinanisols relativ leicht löslich ist, doch ohne Erfolg. 
Das reine Chinanisol ist frisch destillirt ein schwach gelbliches Öl, das aber nach kurzer Zeit auch in gut verschlossenen Gefässen von oben herab grïnlich wird, dann deutlich grtin fluorescirt und endlich eine röthlich-violette Färbung annimmt. Sehr rasch tritt diese Farbenwandlung bei erhöhter Temperatur ein, wesshalb die Destillation in einer indifferenten Atmosphäre anzurathen ist. Es schmeckt brennend, hat einen eigenthümlichen, nicht unangenehmen Geruch, der keine Ähnlichkeit mit dem des Chinolins, eher mit jenem des Anisidins hat, ist in kaltem Wasser sehr schwer löslich, mit den gebräuchlichen Lösungsmitteln sehr leicht mischbar, und bleibt bei $-18^{\circ}$ noch flüssig. Es siedet bei $740 \mathrm{Mm}$. bei 304-305 uncorr. unter geringer Zersetzung; ganz unzersetzt bei $50 \mathrm{Mm}$. Druck bei $193^{\circ}$, bei $35 \mathrm{Mm}$. Druck bei $186^{\circ}$.

Zur Analyse und zur Dichtebestimmung benützte ich im Vacuum destillirte Substanz.

$0 \cdot 2111$ Grm. gaben $0 \cdot 5810 \mathrm{Grm} . \mathrm{CO}_{2}$ und $0 \cdot 1084 \mathrm{Grm}_{2} \mathrm{H}_{2} \mathrm{O}$.

$$
\text { C. } \underbrace{\begin{array}{c}
\text { Berechnet für } \\
\mathrm{C}_{20} \mathrm{H}_{9} \mathrm{NO}
\end{array}}_{\text {H. } .5 \cdot 47} \quad \underbrace{\text { Gefunden }}_{\begin{array}{r}
75 \cdot 06 \\
5 \cdot 90
\end{array}}
$$

Das specifische Gewicht bezogen anf Wasser derselben Temperatur ist bei

$$
\begin{array}{r}
0^{\circ}: 1 \cdot 1665 \\
20^{\circ}: 1 \cdot 1542 \\
50^{\circ}: 1 \cdot 1402
\end{array}
$$

\section{Salze des $p$-Chinanisol.}

Die Salze das Parachinanisols krystallisiren meist sehr gut und sind dadurch besonders charakterisirt, dass die ungefärbten in wässriger Lösung sehr deutlich blau fluoresciren, am schönsten das saure Sulfat, bei dem die Erscheinung ebenso intensiv ist wie beim Chinin. Die Salze geben weiters mit Chlorwasser und Ammoniak die dem Chinin charakteristische Grünfärbung; bei grösserer Concentration ausserdem einen grünen Niederschlag, ganz so wie die Chininsalze selbst. Es kann demnach keinem Zweifel unterliegen, dass diese bekannte Reaction des Chinins 
und Chinidins von dem Chinanisolrest beider Körper verursacht wird. Die Chinanisolsalze geben zum Unterschied vom Anisidin mit Eisenchlorid keine Färbung, mit Platinchlorid und Kaliumbichromat krystallisirte Niederschläge, die in viel kochendem Wasser ganz unzersetzt löslich sind.

Salzsaures Chinanisol $\mathrm{C}_{10} \mathrm{H}_{9} \mathrm{NO} . \mathrm{HCl}+2 \mathrm{H}_{2} \mathrm{O}$ krystallisirt in ungefärbten Prismen, die schon in kaltem Wasser sehr leicht, ebenso im heissen Alkohol schwerer in kaltem, nicht in Äther löslich sind. Concentrirte Salzsäure löst ziemlich reichlich. Am einfachsten erhält man es durch Zufügen von alkoliseher Salzsäure zur warmen Auflösung der Base in Weingeist. Das Krystallwasser entweicht über Schwefelsäure langsam, rasch bei höherer Temperatur, dann aber unter Zersetzung der Trockensubstanz.

1) $0.2011 \mathrm{Grm}$. verloren $0.0162 \mathrm{Grm} . \mathrm{H}_{2} \mathrm{O}$ und gaben $0 \cdot 1359 \mathrm{Grm}$. AgCl und 0.0003 Grm. Ag.

2) 0.2695 Grm. gaben $0.1807 \mathrm{Grm}$. AgCl.

$\underset{\mathrm{HCl} \ldots .17 \cdot 09}{\mathrm{H}_{2} \mathrm{O} \ldots 8 \cdot \mathrm{8} \cdot 43} \quad \underbrace{\frac{1}{17 \cdot 5}}_{\begin{array}{r}17 \cdot 10 \\ 8 \cdot 10\end{array}}$

Neutrales Sulfat. $\left(\mathrm{C}_{10} \mathrm{H}_{9} \mathrm{NO}\right)_{2} \mathrm{H}_{2} \mathrm{SO}_{4}$. In wenig Wasser suspendirtes Chinanisol mit der eben nothwendigen Menge Schwefelsäure in Lösung gebracht, scheidet auf Zusatz von etwas Alkobol weisse Nadeln ab, die in heissem Alkohol leicht, sehr schwer in kaltem löslich sind, in heissem Wasser spielend, in kaltem Wasser nicht sehr leicht zum mindesten schwieriger löslich sind, als die meisten Sulfate von Chinolinderivaten.

Das auf poröser Platte lufttrocken gebrachte Salz verliert über Schwefelsäure langsam, doch constant an Gewicht, die Trocknung wird erst bei $110^{\circ}$ völlig, dabei ist starker Chinanisolgeruch wahmehmbar.

0.2211 Grm. Substanz verloren im Ganzen 0.0876 Grm. und lieferten dann 0.1221 Grm. $\mathrm{BaSO}_{4}$.

Diese Zahlen zeigen, dass die lufttrockene Substanz wasserfreies neutrales Sulfat ist, das bei gewöbnlicher Temperatur langsam, bei erhöhter rasch 1 Mol. Chinanisol verliert und in das saure Sulfat iibergeht. 
Berechnet für

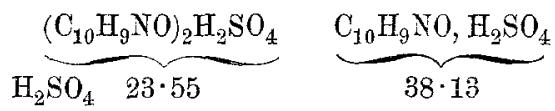

Gefunden

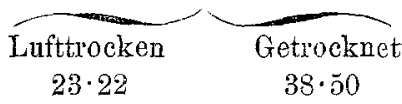

$\mathrm{Saures} \mathrm{Sulfat} \mathrm{C}_{10} \mathrm{H}_{9} \mathrm{NO}, \mathrm{H}_{2} \mathrm{SO}_{4}$. Das schon bei Darstellung des Chinanisols erwähnte Salz ist durch Aufösen in wenig. heissem Wasser und Zusatz des gleichen Volums Alkohol leicht völlig zu reinigen and bildet spröde, weisse Prismen. In Wasser ist es leichter, in Alkohol schwieriger löslich wie das Nentralsalz. Absoluter Alkohol löst sehr schwierig, anch $50 \%$ Weingeist nur sehr wenig, so dass kaltgesättigte wässrige Lösungen durch Alkohol gefällt werden. Das Salz ist krystallwasserfrei.

0.2299 Grm. gaben $0 \cdot 2083$ Grm. $\mathrm{BaSO}_{4}$.

$\underbrace{\text { Berechnet }}_{38 \cdot 43} \quad \underbrace{\text { Gefunden }}_{38 \cdot 10}$

Das weinsaure Salz krystallisirt in Prismen, die in Wasser und Alkohol in der Hitze weit leichter löslich sind als in der Kälte.

Chromat. Die kochende Lösung des Sulfates oder Chlorhydrates scheidet nach Zusatz von Kaliumbichromat lange, seidenweiche Nadeln von beträchtlicher Länge ab, die in kaltem Wasser sehr schwierig gelöst werden. Beim Trocknen an der Laft verlieren die goldgelben Nadeln ihren Glanz and werden etwas bräunlich.

Das Salz ist wasserfrei und lässt sich ohne merkliche Zersetzung bei $100^{\circ}$ trocknen.

$0 \cdot 2473 \mathrm{Grm}$, hinterliessen $0 \cdot 0705 \mathrm{Grm} . \mathrm{Cr}_{2} \mathrm{O}_{3}$.

Berechnet fiur

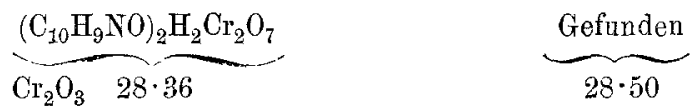

Das Platinsalz bildet goldgelbe, glänzende Prismen und ist in kaltem Wasser schwer, in heissem ziemlich leicht, in vollständig reinem Zustande unzersetzt löslich. Es hat dieselbe Zusammensetzung und alle Eigenschaften des Doppelsalzes, welches seinerzeit mit dem Chinanisol aus $p$-Oxychinolin bereitet wurde. 
$0.2310 \mathrm{Grm}$. verloren bei $100^{\circ} 0.0204 \mathrm{Grm} . \mathrm{H}_{2} \mathrm{O}$ and hinterliessen $0.0558 \mathrm{Grm}$. Pt.

Berechnet für.
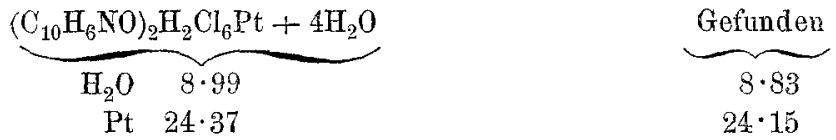

$p$-Chinanisolmethyljodid. Chinanisol mit etwas Äther und überschissigem Jodmethyl vermischt, scheidet bald gelbe Nädelchen ab, deren Vermehrung nach etwa einem Tage beendet ist. Sie sind in Äther nicht, in Wasser und Alkohol in der Kälte schwer, leicht in der Hitze 1öslich. Aus kochendem Alkohol krystallisiren sie wieder in kleinen Prismen, aus heissem Wasser in goldglänzenden spröden, langen Nadeln. Die Verbindung ist krystallwasserfrei, im Capillarrohr erhitzt schmilzt sie bei $235^{\circ}$ unter Gasentwicklung, nachdem von 210 ab Dunkelbraunfärbung eintrat. Im Proberohre erhitzt, zersetzt sie sich unter Ausstossung von scharfriechenden Dämpfen und unter Verkohlung. Geruch nach Methyljodid ist nicht wahrnehmbar, der nach Chinolin äusserst schwach.

0.2528 Grm. gaben 0.1934 Grm. AgJ and 0.0013 Grm. Ag.

Berechnet

J $42 \cdot 18$

Gefunden

$41 \cdot 93$

Das Pikrat löst sich in kochendem Alkohol äusserst schwer, es krystallisirt beim Erkalten in zarten, gelben Nadeln, die beim Trocknen einen schwachen Stich ins Grüne zeigen, gegen $200^{\circ}$ sich braun färben und bei $203-204^{\circ}$ zur bräunlichgelben Flüssigkeit schmelzen. Das Zinn chlorürdoppelsalz krystallisirt in stets vereinzelten Prismen, das Zink dop pelsalz in weichen, nadligen Büschein.

Jodwasserstoffsäure vom specifischen Gewichte 1.96 löst Chinanisol mit Leichtigkeit und schon in der Kälte tritt Geruch nach Jodmethyl auf. Wenn die Lösung etwa eine Stunde im Wasserbade elwärmt, dann eingedampft in Wasser wieder gelöst um dann mit Sodalösung vermischt wird, fällt ein gelblich-krystallinischer Körper aus, der nach all seinen Eigenschaften zweifellos $p$-Oxychinolin ist. Die Bildung des letzteren ist fur die $\mathrm{Natnr}$ des $p$-Chinanisols als $p$-Oxychinolinmethyläther beweisend. 
Herr Dr. v. Jaksch hatte die Güte das Chinanisol in Form verschiedener Salze auf seine klinische Anwendbarkeit zu prüfen. Wie er an anderer Stelle, (Wr. Med. Wochenschr. 1884, Nr. 48), ausfïhrlich mitgetheilt hat, fand er, dass dasselbe schwache antipyretische Eigenschaften besitzt.

\section{Reduction des Chinanisols.}

Verschiedene Versuche das Chinanisol mit Zink in salzsaurer oder schwefelsaurer Lösung zn reduciren, zeigten, dass unter diesen Umständen amorphe, schwer rein darstellbare Körper entstehen, die aller Wahrscheinlichkeit nach condensirte Derivate sind. Der mit Zink und Salzsäure erhaltbare, liefert unkrystallisirbare Salze, ist in Äther schwierig, in Säuren sehr leicht löslich, ebenso in Alkohol and wird durch Eisenchlorid bräunlichgrïin gefärbt. ${ }^{1}$

Anders verläuft die Reduction mit Zinn und Salzsäure. Das Chinanisol nimmt dann glatt Wasserstoff auf und verwandelt sich in ein Tetrahydrochinanisol, das der Kürze halber und wegen der sehr empfindlichen und charakteristischen Griinfärbung, welche diese Base sowie ihre Salze mit fast allen Oxydationsmitteln liefert, Thallin genannt werden soll.

Eine vollständige Reduction des Chinanisols gelingt nur nnter Anwendung von stark überschüssigem Zinn und nicht zu concentrirter Salzsäure, dann aber unter Bildung von wasserlöslichen Producten, die durcb weiter gehende Reduction entstehen und Verluste herbeiführen. Nachdem Thallin und Chinanisol aber ohne Schwierigkeit getrennt werden können, ist es micht nothwendig, die Reduction zu forciren.

Man erhält leicht ein reines Präparat, wenn man die mit etwa der doppelten Menge des theoretisch nothwendigen Zinns bereitete Auflösung in Salzsäure bis zur beginnenden Krystallisation eindampft; nach dem Erkalten sind dann die Zinnchloriirsalze des Thallins und des Chinanisols vollständig abgeschieden.

1 Die von verschiedenen Chemikern mit Zink dargestellten Hydroderivate der Chinaalkaloide dürften auch keine einfachen Hydrokörper, sondern gleichfalls polymerisirte Producte sein. 
Zerlegt man diese mit Schwefelwasserstoff, dampft stark ein und vermischt mit Alkohol, so krystaliisirt das Thallinchlorhydrat ans, das durch vorsichtiges Waschen mit Alkohol endlich Ätheralkohol rein weiss und nahezu frei von Chinanisolsalz erhalten werden, welches in der Mutterlange bleibt.

Umkrystallisiren aus Alkohol-Äther liefert das Thallinsalz dann vollständig rein. Weniger umständlich, doch erst nach einiger Übung mit Sicherheit ausführbar, ist die Trennung durch Zinkdoppelsalze, von denen das des Thallins in Wasser schwieriger löslich ist wie das des Chinanisols. In beiden Fällen hat man erst jene Fractionen als rein anzusehen, die mit Ätzkali oder Ammoniak leicht erstarrende Abscheidungen liefern, die zwischen Papier gepresst kein öl abgeben. Die vorwiegend Chinanisol enthaltenden Mutterlaugen der Chlorhydrate oder Zinkdoppel. salze geben abermals mit Zinn am Wasserbade erwärmt, nene Mengen von Thallin, das in der beschriebenen Weise gereinigt wird u. s. f. Die Totalansbeute ist eine recht befriedigende, nach dreimaliger Reduction erhält man $70-80 \%$ der theoretischen Ausbeute.

Das Thallin aus dem Chlorhydrat ist vollständig rein, das aus dem Zinksalz in der Regel zinkoxydhältig und etwas gefärbt. Die Reinigung gelingt ohne Schwierigkeit durch Überführung in das neutrale Sulfat, dessen concentrirt wässrige Lösung auf Zusatz von Alkohol fast reinweisse Krystalle abscheidet, während die färbenden Verunreinigungen in der Mutterlauge bleiben. Durch Umkrystallisiren aus verdïnntem Weingeist, erhält man sie absolut rein und scheidet dann Ammoniak angenblicklich erstarrende Base ab.

Auch mit Petroleumäther lässt sich die rohe Base rein erhalten, da beim Kochen braungefärbte Substanz vorwiegend ungelöst bleibt, und beim Erkalten zuerst als Öl ausfällt. Der noch warm abgegossene $\ddot{A}$ ther scheidet weisse Krystalle ab.

Das Thallin ist in kaltem Wasser sehr schwer, wenig leichter in heissem löslich, sehr leicht in Alkohol, Äther und Benzol, schwierig in Petroleumäther. Es krystallisirt in dicken Prismen, von schneeweisser Farbe, schmilzt sehr sorgfältig gereinigt bei $42-43^{\circ}$, doch wird auch bei anscheinend tadelloser Substanz der Sebmelzpunkt häufig ein bis zwei Grade zu 
niedrig gefunden. Es siedet bei $735 \mathrm{Mm}$. Druck bei $283^{\circ}$ uncorr. (Thermometer bis $30 \mathrm{im}$ Dampf, Lufttemperatur ' $52^{\circ}$ ) und wenn ganz rein, so gut wie ohne Zersetzung. Mit Wasserdampf verfliissigt es sich nur in geringer Menge.

Die Lösungen des Thallins und seiner Salze, auch die in übersehüssiger Schwefelsäure fluoresciren nicht, Eisenchlorid und viele andere oxydirend wirkende Körper färben dieselben prachtvoll und intensiv smaragdgriin.

Bei Zusatz von wenig Eisenchlorid tritt voribergehend Gelbfärbung nach wenigen Augenblicken aber Dunkelsmaragdgrün ein. Diese Farbe geht beim Kochen in ein Braungrün ïber, dünne Schichten sind dann aber rosenroth. Ein weiterer Zusatz ron Eisenchlorid bewirkt jetzt wieder Grünfärbung, die beim Kochen in Braungrün übergeht, und lässt sich dieser Farbenwechsel durch wiederbolten Eisenchloridzusatz noch oft einleiten, bis endlich der Braungrïnrosadichroismus bleibend wird. Die durch Kochen bewirkte Farbenwandlung tritt bei langem Stehen auch in der Kälte ein.

Dieselben, oder doch ganz ähnliche Erscheinungen geben andere Oxydationsmittel, doch weniger deutlich und nur bei sehr vorsichtigem Zusatz.

Kaliumbichromat gibt die Grïnfärbung nur selten und bei äusserst vorsichtigem Zusatz, da beim geringsten Überschuss die Abscheidung eines braunrothen Chromates eintritt.

Auch Chlorwasser färbt anfangs grün, im Überschusse fällt es ein grünes Harz. Zusatz von Ammoniak vernichtet die Grünfärbung, die dann in eine blassröthliche bis gelbliche übergeht. Das Thallin gibt also die bekannte Chininchlorreaction nicht.

Kaliumnitrit fällt aus wässriger Thallinsalzlösung ein weisses, nicht erstarrendes Öl, das aufZusatz einer Spur Schwefelsäure röthlichgelb wird.

Platinchlorid scheidet anfangs reingelbe Nädelchen $a b$, die aber nach kurzer Zeit unter Grünfärbung der Flüssigkeit metallisches Platin abscheiden.

Silbernitrat färbt unter Silberabscheidung so wie Eisenchlorid.

Salpetersäure färbt die wässrige Lösung nur unter schwer einzuhaltenden Bedingungen grün, meist wird direct rothbraun mit Rosadichroismus gefärbt. 
Festes Thallin, sowie dessen Salze werden von concentrirter Salpetersäure unter lebhafter Erwärmung braungelb gelöst, beim Verdünnen fallen braungelbe Flocken aus.

$\mathrm{Zur}$ Analyse des Thallins eignet sich am besten gefälltes und umgeschmolzenes Material, da das aus Äther, Petroleumäther krystallisirte das Lösungsmittel hartnäckig zurückbehältt.

$0.2090 \mathrm{Grm}$. über Schwefelsäure getrocknet, gaben 0.5616 Grm. CO. und $0.1506 \mathrm{Grm}$. $\mathrm{H}_{2} \mathrm{O}$.

$\underbrace{\begin{array}{c}\text { Berechnet für. } \\ \mathrm{C}_{9} \mathrm{H}_{6} \mathrm{NO} \mathrm{CH}_{3} \mathrm{H}_{4}\end{array}}_{\begin{array}{l}\mathrm{C} \ldots 73 \cdot 62 \\ \mathrm{H} . \ldots 7 \cdot 97\end{array}} \quad \underbrace{8 \cdot 00}_{\begin{array}{r}73 \cdot 23 \\ \text { Gefunden }\end{array}}$

Salzsaures Thallin. $\mathrm{C}_{10} \mathrm{H}_{13} \mathrm{NO}$.HCl. Die Lösung des Thallins in concentrirter Salzsäure scheidet bei langsamer Krystallisation derbe, gut ausgebildete Prismen ab, besonders nach $\mathrm{Zu}$ satz von etwas Weingeist, bei rascher Bildung bekommt man feine, weiche Nadeln. Alkohol löst schwieriger wie Wasser, $\ddot{\text { Ather }}$ gar nicht. Ganz reines Präparat ist am Licht unempfindlich, in der Regel tritt aber nach einiger Zeit schwache Rosafärbung ein. Am deutlichsten sieht man dies an Filtrirpapier (in Folge des Eisengehaltes?), das mit der Lösung getränktwurde. Dasselbe sieht man bei allen anderen Thallinsalzen.

Das salzsaure Thallin ist wasserfrei.

0.3112 über Chlorealcium getrocknet, gaben $0.2178 \mathrm{Grm} . \mathrm{AgCl}$ und 0.0013 Grm. Ag.

$0 \cdot 2396$ über Schwefelsäure getrocknet, gaben $0 \cdot 5162$ Grm. $\mathrm{CO}_{2}$ und $0 \cdot 1528$ Grm. $\mathrm{H}_{2} \mathrm{O}$.

\begin{tabular}{|c|c|c|}
\hline $\begin{array}{l}\text { Berechnet fuir } \\
\mathrm{C}_{10} \mathrm{H}_{13} \mathrm{XO} . \mathrm{HCl}\end{array}$ & \multicolumn{2}{|c|}{ Gefunden } \\
\hline C...66 $60 \cdot 15$ & $59 \cdot 65$ & - \\
\hline Н....7.01 & $7 \cdot 10$ & - \\
\hline $\mathrm{HCl} \ldots 18 \cdot 29$ & - & $17 \cdot 92$ \\
\hline
\end{tabular}

Thallinsulfat $\left(\mathrm{C}_{10} \mathrm{H}_{13} \mathrm{NO}\right)_{2} \mathrm{H}_{2} \mathrm{SO}_{4}+2 \mathrm{aq} \mathrm{krystallisirt} \mathrm{am}$ besten aus verdünntem Weingeist in langen, spröden, weissen Nadeln, ist in der Kälte in Wasser sehr leicht, schwieriger in verdünntem, sehr schwer in absolutem Alkohol löslich; das Krystallwasser entweicht bei $100^{\circ}$ vollständig, die Trockensubstanz ist schwach röthlich gefärbt. 
0.2791 Grm, gaben 0.1411 Grm. $\mathrm{BaSO}_{4}$.

$0.3126 \mathrm{Grm}$. verloren bei $100^{\circ} 0.0242 \mathrm{Grm}$.

Berechnet für

$\underbrace{\left(\mathrm{C}_{10} \mathrm{H}_{13} \mathrm{NO}\right)_{2} \mathrm{H}_{2} \mathrm{SO}_{4}+2 \mathrm{aq}}_{\begin{array}{c}\mathrm{H}_{2} \mathrm{SO}_{4} \ldots .21 \cdot 49 \\ 2 \mathrm{H}_{2} \mathrm{O} \ldots .7 .89\end{array}} \quad \underbrace{\text { Gefunden }}_{\begin{array}{r}21 \cdot 26 \\ 7 \cdot 73\end{array}}$

Thallintartrat ist in Wasser in der Kälte ziemlich schwer löslich, in der Hitze aber äusserst leicht, die heissgesättigte Lösung bleibt beim Erkalten lange übersättigt; Alkohol löst es auch in der Siedhitze schwierig.

Aus Wasser krystallisir' es in dünnen, bei langsamer Krystallisation in dicken, vierseitigen Prismen; aus Alkohol ebenso.

Das Zinnchlorïrdoppelsalz krystallisirt in langen, dünnen Prismen, die stets parallel zu meist dreieckigen Blättern angereiht sind, wie die Rippen einer Blatthälfte; es ist in Wasser nicht sehr leicht, in concentrirter Salzsäure und überschüssigem Zinnchlorür in der Kälte fast gar nicht löslich. Die beschriebenen Nadelaggregate verwandeln sich beim langen Stehen in der Mutterlauge in Blätter desselben Umrisses.

Das Zinkdoppelsalz krystallisirt meist in dünnen Blättern von weisser bis röthlicher Farbe, die concentrisch angeordnet sind, und kann dasselbe sehr leicht von dem in Nadeln krystallisirenden Chinanisolsalz unterschieden werden.

Beim Aufiösen von je 1 Mol. Thallin- und Pikrinsäure in wenig heissem Alkohol entsteht das Pikrat, das lichtgelbe, seidenglänzende Nadeln bildet, die meist concentrisch halbkugelförmig vereinigt, in kaltem Alkohol ziemlich schwierig, in heissem sehr leicht löslich sind. Sie sintern bei $157^{\circ}$ und schmelzen bei $162^{\circ}$.

A c etylthallin. Thallin mit dem mehrfachen Gewicht Essigsäureanhydrid einige Zeit am Rückflusskühler erwärmt, dann in Wasser geworfen, scheidet ein ungefärbtes ätherlösliches Öl ab, das nach einiger Zeit erstarrt, und ans verdünntem Alkohol umkrystallisirt, kleine dicke Prismen bildet. Von den gewöhulichen Lösungsmitteln wird es etwa in demselben Verhältnisse aufgenommen wie das Thallin selbst. Aus einem Gemisch von Äther und Petroleumäther schiesst es in wasserklaren breiten, an- 
scheinend monoklinen Prismen an. Es riecht ähnlich wie Thallin nur weit schwächer.

Der Schmelzpunkt liegt bei $46-47^{\circ}$.

$0 \cdot 1913$ Grm. über $\mathrm{H}_{2} \mathrm{SO}_{4}$ getrocknet, gaben $0 \cdot 4894$ Grm. $\mathrm{CO}_{2}$ und $0 \cdot 1226 \mathrm{Grm}, \mathrm{H}_{2} \mathrm{O}$.

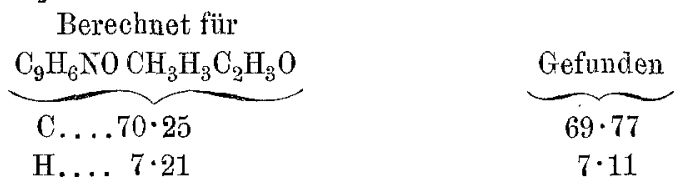

Einwirkung von Brom auf Thallin. Beim Übergiessen von feingepulvertem Thallin mit dem fünffachen Gewicht Brom tritt freiwillige Erwärmung und lebhafte Entwickelung von Bromwasserstoffsäure ein. Nach Verjagen des Broms am Wasserbade bleibt eine röthlichgelbe, nach Brom riechende, feste Masse zurïck, die auch in kochendem Wasser nicht gelöst wird, beim Kochen aber wieder Brom abgibt. Alkohol löst einen Theil mit rothgelber Farbe, während die Hauptmasse mit gelber Färbung: ungelöst bleibt, aus der alkoholischen Lösung erhält man aber nach dem Abdestilliren abermals den gelben, in Alkohol unlöslichen Körper, so dass aller Wahrscheinlichkeit nach das arsprüngliche Reactionsproduct eine additionelle Verbindung: mit Brom ist, die mit Alkohol oder Wasser das freier gebundene Brom verliert.

Die gelbe Substanz ist nur schwach basischer Natur, Eisessig und selbst concentrirte Salzsäure lösen schwierig, beim Verdünnen mit Wasser wird das in Lösung gegangene fast vollständig wieder ausgefällt.

Zur Reinigung wurde in siedendem Xylol gelöst, das bis auf Spuren alles löste, beim Erkalten die Hauptmenge als blassgelbes Pulver wieder abschied, dessen Schmelzpunkt bei $193-194^{\circ}$ liegt.

Zur Analyse 1 und $2 \mathrm{kam}$ der ursprünglich in Alkohol ungelöste, zur Analyse 3 der aus der alkoholischen Lösung gewonnene Antheil in Verwendung.

1) $0.2037 \mathrm{Grm}$. gaben 0.2281 Grm. $\mathrm{CO}_{2}$ und $0.0251 \mathrm{Grm} . \mathrm{H}_{2} \mathrm{O}$.

2) $0.1984 " n 0.2775 " \mathrm{Br} \cdot \mathrm{Ag}$.

3) $0.2573 " n 0.3652, \quad \mathrm{BrAg}$. 
Die Analysen stimmen, wenn auch, was das Brom betrifit, nicht glatt am besten auf eine Zusammensetzung $\mathrm{C}_{10} \mathrm{H}_{6} \mathrm{Br}_{3} \mathrm{NO}$.

$\underbrace{\text { Berechnet }}_{\mathrm{C} . . .30 \cdot 31}$
$\mathrm{H} \ldots . \mathrm{1} \cdot 51$
$\mathrm{Br} \ldots .60 \cdot 50$

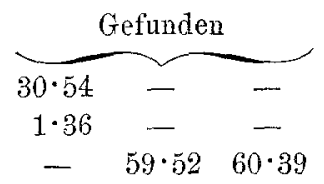

Freies Brom wirkt auf Thallin demnach derart, dass der additionelle Wasserstoff eliminirt und in dem so rückgebildeten Chinanisol $3 \mathrm{H}$ durch $\mathrm{Br}$ ersetzt werden, die Methoxylgruppe aber intact bleibt.

Oxydationsmittel zerstören das Thallin. Eine Lösung von Thallinsulfat lässt auf Zusatz von Kaliumbichromat ein prächtig dunkelbraunrothes Chromat fallen, in der von demselben abfiltrirten Flüssigkeit lassen sich nicht unerhebliche Mengen von Chinanisol nachweisen, dessen Platinverbindung und Chromat dargestellt werden konnten.

Herr Dr. v. Jaksch hat das salzsaure, neutral-schwefelsaure und weinsaure Thallin auf seine klinische Verwendbarkeit geprüft und wie a. a. 0 . ausführlich mitgetheilt ist, gefnnden, dass die Thallinsalze keine Specifica, aber sehr gute Antipyretica sind, die schon in Dosen nnter 1 Grm. beträchtlicbe Temperaturerniedrigungen herbeifuhhren.

Die schwache Wirkung des Chinanisols, die energische des Thallins, sind ein never Beweis für die schon öfter beobachtete Thatsache, dass Chinolin and dessen Derivate durch Aufnahme von Wasserstoff in physiologisch wirksamere Substanzen iibergehen, wie das rom Kärin, das O. Fischer, und Kaïrolin, das

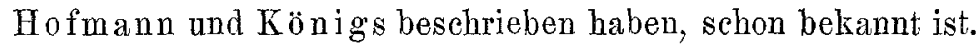

Die energische antifebrile Wirkung des Thallins zeigt aber, dass die Annahme von Filehne: antifebril vermögen solche hydrirte Chinolinderivate $z u$ wirken, deren Imidwasserstoff durch Alkoholradikale ersetzt ist, nicht oder wenigstens nicht für alle Fälle richtig ist.

Filehne hat weiter gefunden, dass hydrürtes Oxychinolin giftig ist, beim Ersatz seines Imidwasserstoffes durch Methyl oder Äthyl seine toxischen Eigenschaften verliere. Auch dieses Zusammentreffen gilt nicht für alle Fälle; denn hydrirtes $p$-Oxy- 
chinolin ist nach Untersuchungen von Jaksch giftig, es verliert aber die gefährliche Wirkung, wenn es bei Ersatz des Hydroxylwasserstoffs vermittelst Methyl den Imidwasserstoff behäIt und in Thallin übergeht.

Das vorliegende Material reicht noch lange nicht hin, um sichere Schlïsse zu gestalten, in welchen Beziehungen chemische Constitution und antipyretische, sowie sonstige Wirkungen organischer Verbindungen stehen, vielleicht ist es aber doch nicht überflüssig darauf hinzuweisen, dass die antipyretischen Eigenschaften vieler Mittel mit ihrer Fähigkeit bei Oxydationsvorgängen alterirt zu werden, im directen Verhältniss zu stehen scheint, wofuir nicht nur in der Chinolinreihe, sondern auch in den Benzolderivaten Beispiele vorliegen.

\section{Methylthallin.}

Ein Versuch, Methylthallin in uiblicher Weise durch Erhitzen einer methylalkoholischen Lösung der Base mit Jodmethyl darzustellen, zeigte, dass jenes hierbei nur in kleiner Menge entstehe, viel Thallin unverändert bleibe, und hauptsächlich das Jodid der quaternären Verbindung gebildet wird. Mannigfache Abänderungen haben hieran wenig zu ändern vermocht, aber einige nicht unwichtige Beobachtungen gestattet.

Von der Ansicht geleitet, dass die Bildung des quaternären Jodids erschwert ist, wenn das früher entstandene jodwasserstoffsaure Methylthallin zum sofortigen Ausfallen gezwungen und damit der weiteren Einwirkung des Jodmethyls grossentheils entzogen werde, habe ich unter anderem Thallin mit der berechneten Menge Jodmethyl in der achtfachen Menge Äther gelöst zusammengebracht, in welchem das erwähnte Salz voraussichtlich nicht löslich ist.

Fast augenblicklich trat Trübung ein und bald schieden sich platte Prismen ab, deren Menge nach 12 Stunden aus 5 Grm. Thallin $5.6 \mathrm{Grm}$. betrug. Die ätherische Mutterlauge abgegossen, schied nach weiteren 24 Stunden 1.5 Crm. nadlige Krystalle ab, und hinterliess dann in einer Schale bei freiwilligem Verdunsten, wobei Geruch nach Jodmethyl wahrnehmbar war, neben kleinen Mengen von Krystallen ein basisches $0 \ddot{1}$, das in verdünnten 
Säuren gelöst, mit Eisenchlorid nicht die grüne Thallinfärbung, sondern eine schöne Rothfärbung gab. Die erste Krystallisation ist ein basisch jodwasserstoffsaures Thallin, die zweite das quarternäre Dimethylthallinjodid, das Öl freies Methylthallin.

Die zuerst erhaltenen platten Prismen wiederholt aus heissem Alkohol krystallisirt, gehen in sehr charakteristische Aggregate von Prismen über, die dem Umfang parallel gestreiften Briefcouverts gleichen. Sie sind in Äther, Benzol und Petroleumäther nicht, schwer in kaltem, leicht in heissem Alkohol löslich. Kaltes Wasser löst fast nicht, heisses theilweise unter Abscheidung eines thallinartig riechenden Öls. Mit Ätzkali oder Soda gekocht, scheiden sie Thallin ab, das nach Schmelzpunkt, Eisenreaction und sonstigen Eigenschaften leicht zu erkennen ist, die kalische Lösung enthält dann Jodmetall.

Der Schmelzpunkt liegt glatt bei 155-156. Die geschmolzene Masse erstarrt beim Erkalten vollständig und ungefärbt. Das Salz ist krystallwasserfrei.

0.3027 Grm. gaben 0.0016 Grm. Ag und 0.1527 Grm. AgJ. $0.2980 \quad n \quad 0.5699 \quad$ " $\mathrm{CO}_{2}$ und 0.1637 Grm. $\mathrm{H}_{2} \mathrm{O}$.

Berechnet fit

$\begin{array}{ll}\overbrace{\ldots .27 \cdot 97}^{\left(\mathrm{C}_{10} \mathrm{H}_{13} \mathrm{NO}\right)_{2} \mathrm{HJ}} & \overbrace{28 \cdot 32}^{\text {Gefunden }} \\ \mathrm{C} \ldots .52 \cdot 86 & 52 \cdot 03 \\ \mathrm{H} \ldots . .6 \cdot 14 & 6 \cdot 14\end{array}$

Die Verbindung ist trotz ihrer eigenthümlichen Lösungsverhältnisse also nichts Anderes wie eine Doppelverbindung von je 1 Mol. Thallin und jodwasserstoffsaurem Thallin. Die Schwerlöslichkeit dieser Verbindung im Zusammenhang mit dem Umstand, dass Methylthallin eine schwächere Base ist, als das Thallin selbst, erklärt den Misserfolg des Versuchs.

Das primär entstandene jodwasserstoffsaure Methylthallin hat sich mit unverändertem Thallin in das beschriebene basische Salz umgesetzt, welches ausfiel, während freies Methylthallin in Lösung blieb, das von Jodmethyl weiter in das quaternäre Jodid verwandelt wurde. Dadurch wurde der $\ddot{A}$ ther relativ ärmer an Thallin, reicher an Jodmethyl, wie die Gleichungen darlegen: 


$$
\begin{gathered}
\mathrm{C}_{10} \mathrm{H}_{13} \mathrm{NO}+\mathrm{CH}_{3} \mathrm{~J}=\mathrm{C}_{10} \mathrm{H}_{12} \mathrm{NOCH}_{3} \mathrm{HJ} \\
\mathrm{C}_{10} \mathrm{H}_{12} \mathrm{NOCH}_{3} \mathrm{HJ}+2 \mathrm{C}_{10} \mathrm{H}_{13} \mathrm{NO}=2\left(\mathrm{C}_{10} \mathrm{H}_{13} \mathrm{NO}\right) \mathrm{HJ}_{+}+\mathrm{C}_{10} \mathrm{H}_{12} \mathrm{NOCH}_{3} \\
\mathrm{C}_{10} \mathrm{H}_{12} \mathrm{NOCH}_{3}+\mathrm{CH}_{3} \mathrm{~J}=\mathrm{C}_{10} \mathrm{H}_{12} \mathrm{NOCH}_{3} \mathrm{CH}_{3} \mathrm{~J}
\end{gathered}
$$

in welchen zusammen auf 3 Mol. Thallin, 2 Molekiile Jodmethyl kommen, und wenn nun schliesslich trotzdem freies und thallinfreies Methylthallin nachweisbar war, kann dies nur in der Art erklärt werden, dass die Bildung des jodwasserstoffsauren Methylthallins leichter erfolgt wie die Entstehung des quarternären Jodids aus Methylthallin, und in dem Masse, als beim Verdunsten des Äthers das überschïssige Jodmethyl entwich, in der Lösung Methylthallin angereichert, das unveränderte Thallin als schwerlösliches basisches Salz abgeschieden wurde.

Es sei noch erwähnt, dass dieselben Producte entstanden, als zur Lösung weniger Äther, oder in verschiedenem Verhältnisse Benzol in Anwendung und die Reaction bei erhöhter Temperatur zum Eintritt kam, nur dass ich dann das quaternäre Jodid in grösster, Methylthallin in kleinster Menge erhielt.

Reichlicher bildet sich Methylthallin beim Erbitzen der Composanten, wenn mit wenig Methylalkohol oder gar nicht verdünnt ist.

$25 \mathrm{Grm}$. Thallin, 50 Methylalkohol und 21.8 Jodmetbyl am Wasserbad zum Kochen erhitzt, riechen nach 1 Stunde deutlich, nach zwei Stunden nur mehr schwach nach Jodmethyl. Nach Verjagung des Alkohols in Wasser gelöst und mit Ätzkali versetzt, schied sich ein Öl ab, das in, der Kälte zam Theil erstarrte. Die Krystalle abgesaugt, dann mit Äther geschüttelt, sind fast ungefärbt und durch wiederholtes Umkrystallisiren aus Alkohol leicht zu reinigen. Der Äther, der das den Krystallen anhängende Öl aufnahm, wurde zum Schütteln der kalischen Mutterlauge benützt. Er nimmt Thallin und Methylthallin auf, die Krystalle sind das Jodid des quaternären Dimethylthallin.

Dasselbe krystallisirt aus Alkohol in meist vereinzelten langen Prismen, aus Wasser in spröden Nadeln von rein weisser Farbe. Sie sind in Alkohol und Wasser in der Hitze leicht, schwer in der Kälte löslich, nicht in Äther, Benzol und Petroleumäther. Kalilauge verändert sie auch beim Kochen nicht, ebenso wenig Salzsäure und Salpetersäure. Im Capillarrobr tritt ohne voran- 
gehende Zersetzung bei 223-24 Verflüssigung ein, wobei stiirmische Gasentwicklung auftritt. Die klare Flikssigkeit erstarrt nach langem Kochen noch nicht. Im Proberohr erhitzt, sublimirt die Substanz, ohne zu eigentlichem Schmelzen zu kommen. Jodmethylgeruch tritt dabei sehr stark auf, die an den kalten Wänden angesetzte krystallinische Substanz ist halbweich. Diese Erscheinungen sprechen dafür, dass bei hoher Temperatur ein Zerfall in Methylthallin und Jodmethyl eintritt.

Die aus Alkohol krystallisirte Substanz ist wasserfrei, die aus Wasser entfällt aber 1 Mol. Wasser, das theilweise schon bei kurzem Liegen an der Luft, rasch bei $100^{\circ}$ entweicht.

1. $0 \cdot 2949 \mathrm{Grm}$. Trockensubstanz gaben $0 \cdot 4866 \mathrm{Grm} . \mathrm{CO}_{2}$ und $0 \cdot 1494 \mathrm{Grm}$. $\mathrm{H}_{2} \mathrm{O}$.

2. $0 \cdot 2687 \mathrm{Crm}$. Trockensubstanz gaben $0 \cdot 1920 \mathrm{Grm}$. AgJ und $0 \cdot 0022 \mathrm{Grm}$. $\mathrm{Ag}$.

\begin{tabular}{|c|c|c|}
\hline \multirow{2}{*}{ 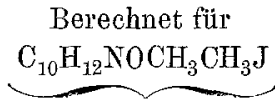 } & \multicolumn{2}{|c|}{ Gefunden } \\
\hline & 1. & 2 \\
\hline$C \ldots \ldots 45 \cdot 14$ & $45 \cdot 00$ & 一 \\
\hline H.... $5 \cdot 64$ & $5 \cdot 62$ & - \\
\hline J. . . .39.81 & - & $39 \cdot 57$ \\
\hline
\end{tabular}

1. $0 \cdot 3099$ Grm. ans Wasser krystallisirt verloren bei $100^{\circ} 0 \cdot 0150 \mathrm{Grm}$.

2. $0 \cdot 2825$
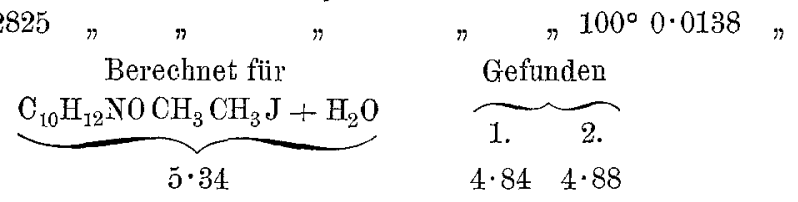

Die wässerige Lösung mit Chlorsilber geschïttelt, scheidet nach dem Filtriren und Eindampfen das in Wasser äusserst leicht lösliche Chlorid in Form schmaler Blätter aus, das weder mit Eisenchlorid, noch einem anderen Oxydationsmittel eine Färbung gibt. Platinchlorid fällt einen gelbröthlichen Niederschlag, der aus kochendem Wasser in lebhaft glänzenden Blättchen von orangerother Farbe auskrystallisirt, die krystallwasserfrei sind.

Analyse der bei $100^{\circ}$ getrockneten Substanz:

0.2708 Grm. gaben 0.0672 Grm. Pt, 0.3550 Grm. $\mathrm{CO}_{2}$ und 0.0961 Grm. $\mathrm{H}_{2} \mathrm{O}$. 


$\begin{array}{cr}\begin{array}{c}\text { Berechnet für } \\ \left.\left(\mathrm{C}_{9} \mathrm{H}_{6} \mathrm{~N}(\mathrm{OCH})_{3}\right) \mathrm{H}_{3} \mathrm{CH}_{3} \mathrm{CH}\right)_{2} \mathrm{Cl}_{6} \mathrm{Pt}\end{array} & \underbrace{\text { Gefunden }} \\ \mathrm{H} \ldots \ldots 36 \cdot 36 & 3 \cdot 95 \\ \mathrm{Pt} \ldots . .24 \cdot 54 & 24 \cdot 81\end{array}$

Methylthallin. Der Äther, mit dem das quaternäre Jodid von Öl befreit und der zum Ausschütteln des kalisehen Filtrates benitzt wurde, enthält Methylthallin nnd Thallin gelöst. Das Basengemenge gibt eine rothgrüne Eisenreaction.

Die Trennung beider Basen durch irgend ein Salz geht schwierig vor sich; zwar lässt sich die Hauptmenge des Thallins abscheiden, wenn man in Salzsäure löst und eindampft, da es zuerst auskrystallisirt und Methylthallin von wenig Thallin trennen, da sein saures Sulfat besser krystallisirt, doch sind diese Trennungen sehr mühsam, da die Gemenge im Allgemeinen sehr langsam Krystalle ansetzen, die erst nach wiederholtem Umkrystallisiren ganz rein zu erhalten sind.

Am einfachsten und raschesten ist es, die ätherische Lösung fractionell mit verdünnter Schwefelsäure zu schütteln, die anfänglich bloss Thallin, sehliesslich nur Methylthallin entzieht. Die mittleren Fractionen der Salzlösung geben bei fractionellem Zusatz von Kalilange und Schütteln mit $\ddot{A}$ ther an diesen wieder vorerst Methylthallin, später erst Thallin ab, so dass beim Arbeiten mit titrirten Lösungen in kurzer Zeit fast vollständige Anflösung des Gemisches erzielt werden kann, wenn man die den reinen Substanzen eigenthtimalichen Eisenchloridreactionen als Massstab der Reinigung festhält.

Das reine Methylthallin ist ein nicht erstarrendes, dickes Öl, das frisch destillirt nahezu ungefärbt ist, allmälig aber eine röthlichbraune Färbung annimmt. Es riecht eigenthümlich, rom Thallin ganz verschieden, und siedet bei $277-278.5$ uncorr.

Die Auflösung in verdïnnter Salzsätrre wird durch einen Tropfen Eisenchlorid dunkel kirschroth gefärbt, beim Kochen wird die Farbe anfangs dunkler, später aber licht röthlichgelb. Der den. Thallinsalzen eigenthiumliche rothe Dichroismus ist dabei nicht wahrzunehmen. Platinchlorid fällt einen harzigen Niederschlag, der beim Kochen nnter Platinabscheidung zersetzt wird. Chlorwasser färbt rothgelb, anf Zusatz von Ammoniak 
fällt ein weisser Niederschlag aus, die Flüssigkeit ist dann dunkel pfirsichblutroth.

Das salzsaure Salz ist in Wasser sehr leicht löslich, kann aber in gut ausgebildeten dicken Prismen gewomnen werden, die nicht hygroskopisch sind.

Auch das saure Sulfat krystallisirt in gut ausgebildeten dicken Prismen, am besten, wenn die stark concentrirte wässerige Lösung mit Alkohol überschichtet wurde. Es ist in Wasser sehr leicht, in Alkohol um so schwieriger löslich, je absoluter er ist, and kann auch aus stark gefärbten Lösungen sehr leicht vollstänơig farblos erhalten werden.

Das Salz ist krystallwasserfrei, beim Trocknen färbt es sich schwach röthlich.

1. $0 \cdot 2780 \mathrm{Grm}$. gaben $0 \cdot 4941 \mathrm{Grm} . \mathrm{CO}_{2}$ und $0 \cdot 1445 \mathrm{Grm} . \mathrm{H}_{2} \mathrm{O}$.

2. $0.2510 " 0.2154$ " $\mathrm{BaSO}_{4}$.

\begin{tabular}{|c|c|c|}
\hline \multirow{2}{*}{$\begin{array}{c}\text { Berechnet für } \\
\mathrm{C}_{9} \mathrm{H}_{6} \mathrm{~N}\left(\mathrm{OCH}_{3}\right) \mathrm{H}_{3} \mathrm{CH}_{3}, \mathrm{H}_{2} \mathrm{SO}_{4}\end{array}$} & \multicolumn{2}{|c|}{ Gefunden } \\
\hline & 1. & 2. \\
\hline C.....48.00 & $48 \cdot 47$ & - \\
\hline H...... $6 \cdot 18$ & $5 \cdot 80$ & - \\
\hline $\mathrm{H}_{2} \mathrm{SO}_{4} . .35 \cdot 64$ & - & $36 \cdot 09$ \\
\hline
\end{tabular}

Mit Methyljodid vermischt, erstarrt das Methylthallin allmählig zu einem Brei weisser Prismen, die vollständig identisch mit dem oben beschriebenen quarternären Salz sind.

\section{Athylthallin.}

Während Thallin mit Methyljodid die tertiäre Base nur in untergeordnetem Masse bildet, liefert es mit Äthyljodid dieselbe als Hauptproduet.

Thallin löst sich in der für 1 Mol. berechneten Menge Jodaethyl fast vollständig unter Temperaturerniedrigung, beim Erwärmen am Wasserbad tritt aber lebhafte Reaction ein, die nach viertelstündiger Dauer beendet ist. Der in Wasser gelöste Kolbeninhalt, bestehend aus einer zähen Masse, in der Krystalle eingebettet sind, scheidet auf Zusatz der berechneten Menge von Ätzkalilösung zwei Ölschichten ab, von denen die obere freieBase, die untere das quarternäre Jodid ist, beim Durchschiitteln mit gewöhnlichem, also alkoholhältigem Äther verschwindet die letztere. 
Die ätherische Lösung, die neben Äthylthallin unverändertes Thallin enthält, wird durch fractionelles Sättigen in derselben Weise auf reine Base verarbeitet wie es beim Methylthallin beschrieben ist, auch die Eisenchloridreaction ist hier ganz dieselbe.

Das reine Äthylthallin ist dem Methylthallin äusserst ähnlich, wie dieses dickflussig, es siedet bei $287-287 \cdot 5^{\circ}$ uncorr. unter geringer Zersetzung; unter Abhaltung von Luft destillirt, ist es gelb, nach einiger Zeit wird es aber unter allen Umständen hellbraunroth. In Wasser ist es nicht, in Alkohol, Äther und Mineralsäuren sehr leicht, schwierig in organischen Säuren löslich. Die Lösung in Essigsäure scheidet es beim Verdunsten wieder ab.

$0.2521 \mathrm{Grm}$. gaben $0.6948 \mathrm{Grm} . \mathrm{CO}_{2}$ und $0.2031 \mathrm{Grm} . \mathrm{H}_{2} \mathrm{O}$. Berechnet für
$\underbrace{\mathrm{C}_{10} \mathrm{H}_{18} \mathrm{NO}^{\mathrm{N}} \mathrm{C}_{3} \mathrm{H}_{5}}$
Gefunden
C.... . $75 \cdot 39$
$75 \cdot 16$
H..... 8.90
$8 \cdot 95$

Die Salze des Äthylthallins sind meist sehr hygroskopisch, in Wasser äusserst löslich, und krystallisiren schwierig. Es gelang das Tartrat und Chlorhydrat in guten Krystallisationen zu erhalten, ersteres durch Zusatz einer Lösung von Weinsäure in Äther, letzteres durch Einleiten von Salzsäuregas in die ätherischeLösung der Base; auch ein Pikrat ist in fester Form zu erhalten, das anfangs harzig ausfällt, mit Petroleumäther übergossen aber nach einigen Tagen krystallinisch erstarrt.

Das salzsaure Äthylthallin fällt beim Einleiten von Salzsäuregas in Äther anfänglich weich, erst später trocken krystallinisch aus, und selbst dann, wenn mit den gewöhnlichen Hilfsmitteln von Wasser und Alkohol gereinigter, sogenannt absoluter Äther gewonnen wurde. Aus Petroleumäther schied es sich stets halbweich aus, auch wenn derselbe iiber Ätzkalk und Natrium destillirt war. Das ausgefällte Salz muss, nachdem der Äther abgegossen wurde, angenblicklich über Schwefelsäure ins Vacuum gebracht werden, soll es nicht grösstentheils zerfliessen. Die im Vacuum getrocknete Salzmasse lässt sich dann an trockener Luft, ohne merklich feucht zu werden, aufbewahren. 
Ganz unmerkliche Quantitäten von Wasser genügen, das Salz zum Zerfliessen zu bringen, so dass die Substanz zwischen den Fingern gerieben angenblicklich flùssig wird, ebenso beim Anhauchen.

Aus Wasser krystallisirt es nach langem Stehen in grossen, tafelartigen Krystallen, die zwar auch in Alkohol äusserst leieht löslich sind, aber bei raschem Arbeiten doch ohne merklichen Verlust durch Anrühren mit Weingeist und Absaugen von der kleinen Menge gefärbter Mutterlauge zu trennen sind.

Sowohl das aus Äther gefällte, wie das aus Wasser krystallisirte Salz ist wasserfrei.

0.2438 Grm. im Vacuum getrocknet, gaben $0 \cdot 1470 \mathrm{Grm}$. AgCl und $0.0022 \mathrm{Grm}$. Ag.

$$
\underbrace{\begin{array}{c}
\text { Berechnet für. } \\
\mathrm{C}_{10} \mathrm{H}_{12} \mathrm{NOC} \mathrm{C}_{2} \mathrm{H}_{5} \mathrm{HCl}
\end{array}}_{\mathrm{HCl} \ldots .15 \cdot 97} \overbrace{15.57}^{\text {Gefunden }}
$$

Die wässerige Lösung des Salzes gibt mit Eisenchlorid, mit Chlorwasser und Ammoniak ganz genau dieselben Reactionen wie die Salze des Methylthallins. Platinchlorid wird aber sehwieriger und in der Kälte fast gar nicht reducirt. Concentrirte Salzlösungen lassen auf Zusatz von Platinchlorid ein allmählig erstarrendes Öl fallen verdünnte scheiden allmählig braungelben Niederschlag ab.

$\ddot{A}$ thylthallinäthyljodid. Die wie oben unter $\ddot{A}$ thylthallin erwähnt wurde, vom ätherisehen Auszuge des Thallins und Äthylthallins getrennte wässerige Flüssigkeit enthält neben Jodkalium das genannte Additionsprodnet.

Beim Concentriren scheidet es sich als in der Kälte erstarrendes braunes Öl ab, das durch Umkrystallisiren aus Alkohol schwierig von beigemengtem Jodkalium getrennt werden kann, am besten noch durch fractionelles Fällen der alkoholischen Lösung mit Äther. Das Salz ist in reinem Wasser leicht, schwierig in jodkaliumhältigem, leicht in Alkohol, nicht in Äther löslich und bildet meist weisse Nadeln, bei langsamer Verdnnstung aus Alkohol aber grosse Krystallkörner.

Es schmilzt nach vorhergehendem Erweichen bei 131-33 unter Gasentwicklung, im Destillationskölbchen erhitzt, zerlegt 
Skraup.

es sich sehr glatt in $\ddot{A}$ thyljodid und Äthylthallin die fractionell aufgefangen werden können. ${ }^{1}$

Selbstverständlich entsteht dieselbe Verbindung aus Äthylthallin und Jodäthyl. Die beiden Körper reagiren aber sehr langsam, so dass nach zwölfstiindigem Stehen nur wenige Nadeln des Additionsproductes ausgefallen sind, und auch nach fünfstundigem Erhitzen am Wasserbad ist die Vereinigung noch nicht vollständig.

Beim Schuitteln mit Chlorsilber geht das Jodid in das Chlorid über, das in Wasser sehr leicht lösliche Blätter liefert, ein in kaltem Wasser schwer, in heissem wenig leichter lösliches Chloroplatinat in Form orangegelber kleiner Nädelchen gibt, das wasser frei krystallisirt.

0.2622 Grm. hinterliessen 0.0604 Grm. Pt.

Berechnet für

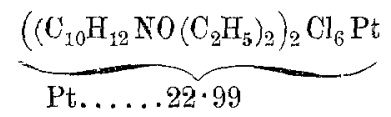

$$
\underbrace{\text { Gefruden }}_{23 \cdot 03}
$$

Das Chlorid bleibt mit Eisenchlorid oder mit Salpetersäure vermischt, vollständig farblos, mit Chlorwasser und dann mit Ammoniak vermischt, bekommt es aber eine kaum wahrnehmbare Grünlichfärbung.

\section{Benzylthallin.}

Moleculare Mengen von Thallin und Benzylchlorid eine Zeit lang erhitzt, schmelzen zu einer dicken Masse, in der ein quaternäres amorphes Chlorid, unverändertes Thallin, und eine Base nachweisbar sind, die mit Eisenchlorid die dem Methyl- und Äthythallin charakteristische Rothfärbung liefert, demnach das Benzylthallin sein duirfte.

Die vorliegenden Resultate gestatten bezüglich der Constitution des Chinins eine Ansicht auszusprechen, die, wenn auch nicht streng bewiesen, doch durch Wahrscheinlichkeitsgründe gestütat werden kann.

1 In Folge dieses Umstandes wäre es wohl die bequemste Darstellung des Äthylthallins und wahrscheinlich anderer ähnlicher tertiärer Basen, wenn direct auf die quaternäre Verbindung hingearbeitet, und diese dann durch Erhitzen in das tertiäre Amin und Jodmethyl zerlegt wïrde. 
Chinin und die anderen wichtigeren Chinaalkaloide enthalten so viel Wasserstoff, dass die Chinolin- respective Pyridinreste, die man in denselben anzunehmen gezwungen ist, nicht als solche sondern nur in hydrürter Form vorhanden sein können.

Vorwiegend physiologische Gründe haben nun zu der Annahme geführt, daśs im Chinin, Cinchonin etc. an einem hydrürten Pyridinring (respective Chinolin) der Imidwasserstoff durch Methyl ersetzt ist, dabei ist aber nur der Rest der Alkaloide in Betracht gezogen worden, der bei der Oxydation nicht Cinchonin-, respective Chininsäure liefert.

Experimentell ist diese Anschaung überhaupt und insbesondere im letzten Punkte nun in gar keiner Art bewiesen; hydrürtes Chinolin gibt mit Oxydationsmitteln Chinolin, Thallin geht in $p$-Chinanisol über und es könnte ja immerhin möglich sein, dass jener Theil des Chinins z. B., der bei der Oxydation Chininsäure liefert, im Chinin redueirt und im Imidwasserstoft durch $\mathrm{CH}_{3}$ ersetzt sei, bei der Oxydation aber vorerst Methyl und Wasserstoff abgespalten und dann erst die Chininsäure gebildet wird.

In diesem Falle wäre aber das Chinin ein Derivat des Methylthallins, das ebenso wie alle Alkylderivate des Thallins mit Eisenchlorid, Salpetersäure etc. sehr intensive Färbungen gibt, von Kaliumbichromat, Platinchlorid, Silbernitrat sehr leicht oxydirt wird, was bekanntlich beim Chinin und Chinidin nicht zu beobachten ist, und desshalb scheint mir die übliche Annahme, dass der Chinanisolrest im Chinin (und Chinidin) als solcher und nicht hydrürter vorhanden sei, allerdings die richtige.

Eine ähnliche Überlegung führt za demselben Schluss für den Cinchoninsäure liefernden Chinolinrest des Cinchonins and Cinchonidins.

Dafür spricht noch ein anderer Umstand. Chinin mitZion und Salzsäure reducirt, geht in eine amorphe Base über, deren Salze äusserst löslich und nicht zum Krystallisiren zn bringen waren, ausserdem schon an der Luft sich langsam zersetzen und dabei immer wieder Chininsalze abscheiden. Es ist mir nicht gelungen, irgend eine Verbindung in analysenfähige Form zu bringen, doch dürfte sicher stehen, dass eine Hydroverbindung des Chinins vor- 
liegt, die schon durch die Luft zum Theil zu Chinin rïckoxydirt wird. Dieselbe zeigt nun vor Luft geschützt dargestellt, mit Eisenchlorid intensiv und genau alle die charakteristischen Färbungen wie sie dem Thallin eigenthümlich sind, während der Luft länger ausgesetzte Substanz nur schwache Reaction gibt. Auf oben ausgesprochener Ansicht fussend, ist diese Übereinstimmung leicht verständlich, da ja der Chinanisolrest im Chinin ebenso reductionsfähig sein kann, wie das Chinanisol selbst. Andererseits kommt noch in Betracht, dass das Chinanisol die charakteristischen Reactionen des Chinins: Flnorescenz insbesondere in saurer Lösung, dann Grünfärbung mit Chlorwasser und Ammoniak gibt, das Thallin und die tertiären Thalline aber nicht.

Nicht so bestimmt, aber doch ziemlich unwahrscheinlich macht obige Überlegung die auch schon geäusserte Vermuthung, dass der $N$ des Cinchonin-chinolins, respective Chinin-chinanisols die Verbindung mit dem anderen Alkaloidrest vermittle.

Diesennegirenden Betrachtungen möchte ichnoch eine andere anschliessen. Die Entstebung ron Cinchoninsäure aus Cinchonin wird häufig erklärt, indem man im Chinolinkern eine Methylgruppe annimmt, die bei der Oxydation in die Carboxylgruppe übergehen soll.

Bedenkt man nun, dass alle methylirten Chinolinderivate nur äusserstschwierig und stets unter theilweiser Zerstörung zu Carbonsämren oxydirt werden, die Oxydation des Cinchonins aber ziemlich leicht erfolgt und sehr gute Ausbeute an Cinchoninsäure liefert, so wird die Wahrscheinlichkeit jener Ansicht über Constitution des Cinchonin's (und der anderen drei bekannteren Chinabasen) eine sehr geringe.

Die vor einigen Jahren von mir in Aussicht gestellte Untersuchung der aus Cinchonin und Chinin neben Cinchonin-, respeetive Chininsäure entstehenden syrupösen Oxydationsproducte ist nach Überwindung vieler Schwierigkeiten so weit gediehen, dass eine Trennung der Bestandtheile des Syrups gelnngen ist. Die mithseligen Methoden, die zur Trennung und Reindarstellung nothwendig sind, verzögern aber das Fortschreiten der Arbeit und ist die Untersuchung noch lange nicht beendet; ich hoffe indessen in kurzer Zeit den ersten Theil derselben abzuschliessen. 